\title{
Medical Expert Support Tool (MEST): a person-centric approach for healthcare management
}

\author{
Carlos Cavero ${ }^{1}$, Juan Mario Rodríguez ${ }^{1}$, Rosana Valle ${ }^{1}$, Alberto Rugnone ${ }^{2}$, \\ Francesco Foresti ${ }^{2}$, Elena Tamburini ${ }^{2}$, Cristiano Paggetti ${ }^{2}$, John Gialelis ${ }^{3}$, Petros \\ Chondros $^{3}$, Christos Panagiotou ${ }^{3}$, Maitena Ilardia ${ }^{4}$, Iñaki Eguia ${ }^{4}$, Paolo Emilio Puddu ${ }^{5}$ \\ ,Mitja Luštrek ${ }^{6}$ \\ ${ }^{1}$ ATOS, Albarracín 25, 28037 Madrid, Spain \\ \{carlos.cavero, juan.rodriguezp, rosana.valle\}@ atosresearch.eu \\ ${ }^{2}$ I+ s.r.l., pz Puccini 36 \\ \{a.rugnone, f.foresti, e.tamburini, c.paggetti\}@i-piu.it \\ ${ }^{3}$ Industrial Systems Institute, Stadiou str, 26504, Platani, Patras, Greece \\ \{gialelis, cpanag, pchondros\} @ece.upatras.gr \\ ${ }^{4}$ Fundación Tecnalia R\&I, Paseo Mikeletegui 2, 20009 San Sebastián, Spain \\ \{maitena.ilardia, inaki.eguia\}@tecnalia.com \\ ${ }^{5}$ University of Rome "La Sapienza", Viale del Policlinico 155, Rome 00161, Italy \\ paoloemilio.puddu@uniroma1.it \\ ${ }^{6}$ Jožef Stefan Institute, Jamova cesta 39, 1000 Ljubljana, Slovenia \\ mitja.lustrek@ijs.si
}

\begin{abstract}
The Medical Expert Support Tool (MEST) is aimed at helping the clinician in recognizing risk factors in the patient status by offering a multiparametric overview, and by highlighting the individual situation using meaningful colors (green, yellow and red) in order to compare the person physiological parameters with the computed profile. The medical professionals configure the conditions (relevant parameters, thresholds, rules and alerts) setting the values to the decision support modules and receiving the risk assessment results. Finally, interventions should be done depending on the evaluation of the patient. The tool has been designed along with the clinician involved in the project and it will be fully tested and evaluated during the observational study (100 patients) starting on June 2012.
\end{abstract}

Keywords: Medical Expert Support Tool, risk assessment, healthcare, personcentric, user-centered design 


\section{Introduction}

Chronic diseases, such as heart disease, stroke, cancer, chronic respiratory diseases and diabetes, are by far the leading cause of mortality in the world, representing $63 \%$ of all deaths [1]. The quality of life of such patients is severely affected by complications and continuous visits to hospitals to monitor physiological parameters increasing thus healthcare and social costs.

The Medical Expert Support Tools (MEST) is a set of UI-based tools built on top of Computer Based Clinical Decision Support System (CDSS) of the system. They have the purpose to help the medical professionals to detect risk from chronic disease monitoring and subsequently to manage the risk in terms of assessment and intervention to mitigate the dangerous situation related to the detected risk factor.

Clinical decision support systems, CDSS [2], must be integrated with a health care organization's clinical workflow, which is often already complex. MEST does not add more complexity on top of preexistent clinical systems but is fully integrated with them, whether they are based on EHR [3] or not.

Similar existing tools as QRISK2 [4] cardiovascular risk score (designed to identify people at high risk of developing CVD) and ARRIBA [5] risk calculator (designed to calculate the risk of experiencing a heart attack or a stroke) are not focused in the continuum of care of the patients.

MEST provides the medical professionals with a multiparametric view and an overall status of the patient indicating the risk factors with meaningful colors (green, yellow and red). The assessment of the person's risk based on the analysis of the information already stored in the system offers the doctor a clear insight of the current situation of the individual. The detection of critical situations will activate the feedbacks to the patient and to the clinicians.

The solution provided to the medical professionals has followed a person-centric approach allowing the patients acceptance through the non-invasive and personalized risk profile. To reach the goals, a clinical expert workflow, which describes the steps of the system, has been designed by interviewing the medical professionals involved. The general schema of the workflow developed is shown in Fig 1. 


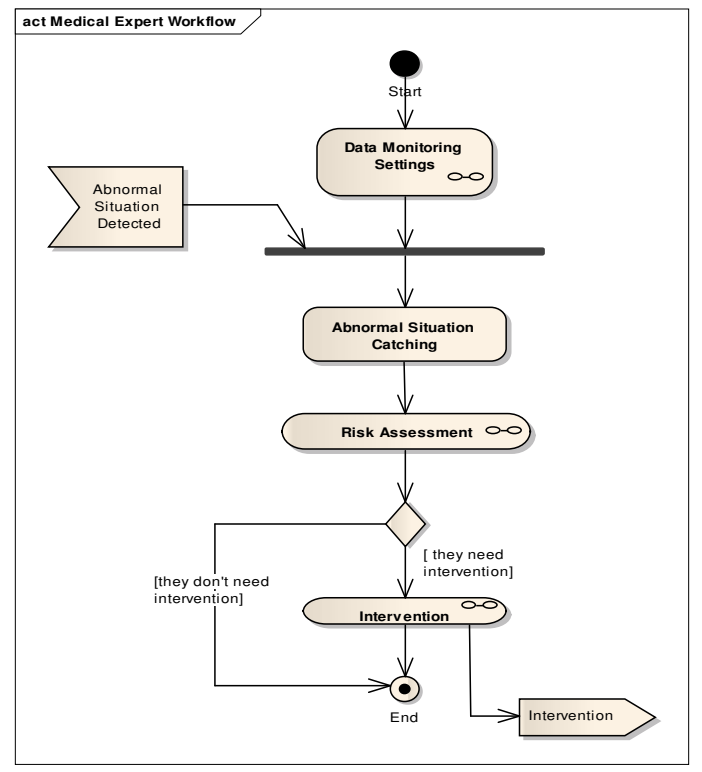

Fig. 1. MEST workflow

The medical professionals access the MEST UI via https (internet/intranet) and it consists of three parts:

Data Monitoring Settings. It sets the inputs to the corresponding decision support system modules in order to configure the system

- Prioritized Parameters. Stand-alone application which finds relevant parameters to be measured.

- Personalized Monitoring Settings. Parameters, thresholds, rules and feedbacks configuration by the clinician.

Risk Assessment. This module receives the information from the Decision Support modules in order to give the proper risk factor to the doctors.

Interventions. It permits the doctor to manually (or with automatically generated suggestions) interact with the user once all the information coming from the Decision Support System is analyzed.

\section{Person-centric designed approach}

The User-centred Design methodology is a user- and task-oriented requirement engineering methodology that integrates system level requirements and usability requirements in a holistic elicitation and specification approach.

This methodology follows a User-centred Design (UCD) method and all the stakeholders are to be involved in the capture of the user requirements and integrate Requirement Engineering and Usability Engineering to achieve a better user-centred approach. It includes the definition of user-centred techniques to elicit non-functional 
requirements in the AAL context and will ensure that the methodology has considered all potential users and stakeholders interests, needs and wants and has adequately involved them in all phases of development (user needs, specifications, design and development, interactive testing, dissemination, exploitation and training).

The UCD guidelines herein are to be used for User and Stakeholders Groups profile identification, User Requirements extractions, applications development, realization of pilot plans and verification activities in the User-centred Design methodology.

ISO 9241-210:2010 [6] has been followed to design the methodology process model to help designers to fulfil the goal of a product engineered for its intended users. This standard provides requirements and recommendations for human-centred design principles and activities throughout the life cycle of computer-based interactive systems.

Several phases have been proposed for the development and validation process of the solution, (i) Analysis Phase, (ii) Design Phase, (iii) Implementation Phase and (iv) Deployment Phase.

The stakeholder collaboration was crucial. The doctors involved in the project helped in the requirements elicitation and also during the design and the implementation phase. The first unit tests have been carried out by the cardiologist in order to improve the tool in an iterative process.

\section{Medical Expert Support Tool (MEST)}

The MEST workflow starts with the pre-configuration of the systems, which means the selection of the parameters for each patient. The Prioritized Parameter (PP) component gives the doctor new findings in order to take into account more information. The second step consists in the selection of the parameters and thresholds needed for the decision support module depending on the patient's health.

The risk assessment component is divided into three sub-components using three different approaches. The expert system sub-component incorporates existing medical knowledge. The machine learning sub-component learns the relation between the parameters characterizing the patient's health and his/her risk using an Artificial Neural Network. The anomaly detection sub-component detects anomalies in the values and relations between the values of the parameters characterizing the patient's health, using the Local Outlier Factor algorithm.

Once all the above mentioned parameters are set, the risk assessment starts and the status of the patient is shown evaluating the risk factors. Finally the required intervention is made depending on the risk detected.

\subsection{Prioritized Parameters}

This component focuses on the identification of monitoring parameters that are characteristic for CHF patients and have great impact on their health status. In order to follow such a personalized approach data mining techniques have been implemented by employing pattern mining methods. The module is capable of 
extracting previously unknown patterns through an automatic analysis on large amount of gathered medical data and discovering associations and possible hidden relations between extracted parameters from various clinical data sources.

The proposed mining pattern follows the definition of an association rule, $X=>Y$, where $X, Y \in I$ are called antecedent (Left Hand Side, LHS) and consequent (Right Hand Side, RHS), with being a set of $\mathrm{n}$ binary attributes called itemset. Each item of the rule can be a parameter name or a combination of parameters with correspondent ranges. For example, rule ST $[-2,-0.5]=>$ STATUS [normal] can be read as "ST parameter that its value ranges from -2 to -0.5 implies a normal STATUS".

The input to the module is a labeled dataset, converted in readable format by discretization functions mainly, which may come from a classification mechanism or from medical experts. Since the generated rules are semantically weak, there is no guarantee that these rules implied any deeper relationships. The key idea is to use only the "interesting" association rules. This choice is based on the use of objective measures which can filter and sort automatically large quantity of generated rules.

The medical expert through the use of a GUI is able to further process results taking advantage of the options listed below:

- Prune in advance the number of the rules to be generated by configuring (manually or automatically) minimum support and minimum confidence values accordingly.

- Discard rules that show independency between RHS and LHS of a rule (manually or automatically). Compare all results using the overall view in the GUI.

- Sort the rules per different metric value focusing each time in different property regarding the relationship between the attributes of the rule.

- Compare the strength of rules given the same antecedent and more specific when the antecedent is the "ABNORMAL" status of the patient. This kind of enhancement aims to determine the strongest direction of the rule.

The medical expert can observe and sort the rules depending on a metric that evaluates its strength (including confidence, lift, leverage and conviction) and can evaluate the patterns in order to reconsider past or future decisions that need to be taken for the patient's monitoring and treatment. As the amount of medical data increases the performance of the algorithm is enhanced. However it is important to underline that this application has an advisory role for the medical expert.

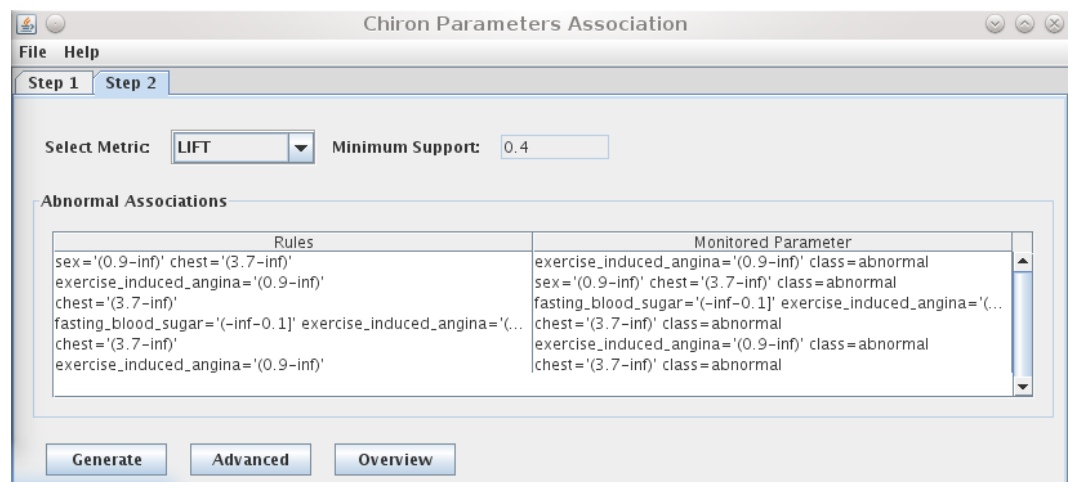

Fig. 2. Screenshot of the priority parameters module 


\subsection{Monitoring Settings}

The Monitoring Settings is the system pre-configuration in order to prepare the decision support modules and set the corresponding boundary values (high and low). After receiving the relevant parameters from the Prioritized Parameters component the medical professionals set the parameters, rules, thresholds and alerts in order to configure the Risk Assessment module. All the elements get the data from a common repository. Once the clinician has set the parameters, he/she has to define a set of rules in order to send personalized feedback to the patients. All the configuration thresholds can be computed dynamically (intelligent system) based on past experience of certain diseases, but it also includes manual interaction, it means, the doctor could set the values using the GUI.

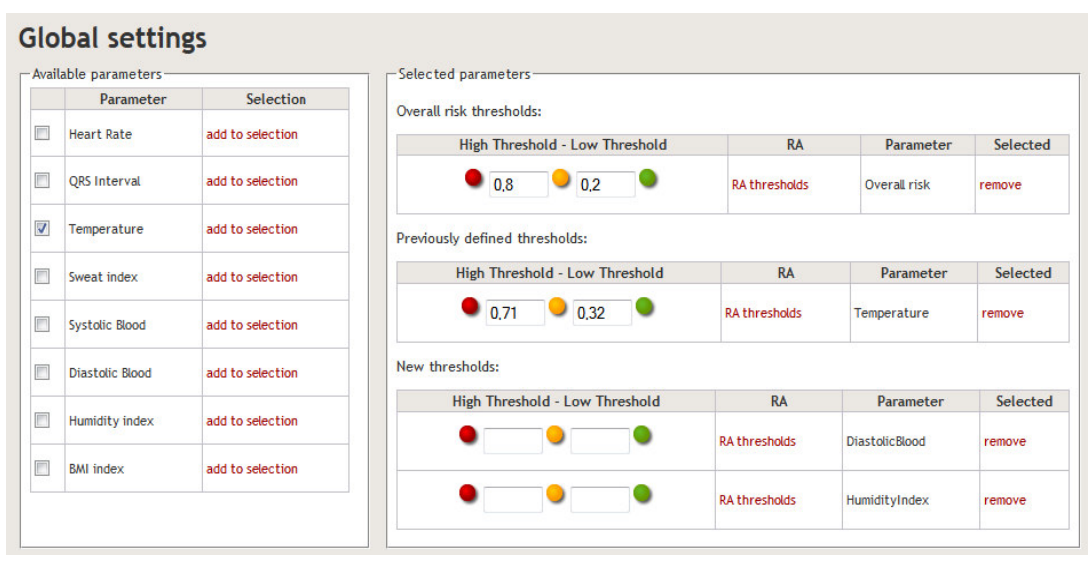

Fig. 3. Monitoring Settings GUI

The Monitoring Settings module describes the parameters which, automatically or manually guide the patient profile and the rules-set configuration to trigger the corresponding feedbacks to the clinician and patients. The Medical Expert Workflow sets up the inputs (values) to the other components and could also receive the corresponding outputs (suggestions).

Once the modules have been configured the risk assessment process is started and the tool guides the doctor in knowing the factors which influence the patient status.

\subsection{Risk Assessment}

The risk assessment procedure is the central part of the workflow where the clinician is expected to review the clinical status of his/her patients and subsequently schedule an intervention if needed.

This procedure is preceded by the "Abnormal Situation Catching" step of the workflow. This is where the clinician is presented with the list of his/her patients (Fig 4). Every patient is associated with a brief evaluation of his/her overall risk factor by means of a green/yellow/red mark. 


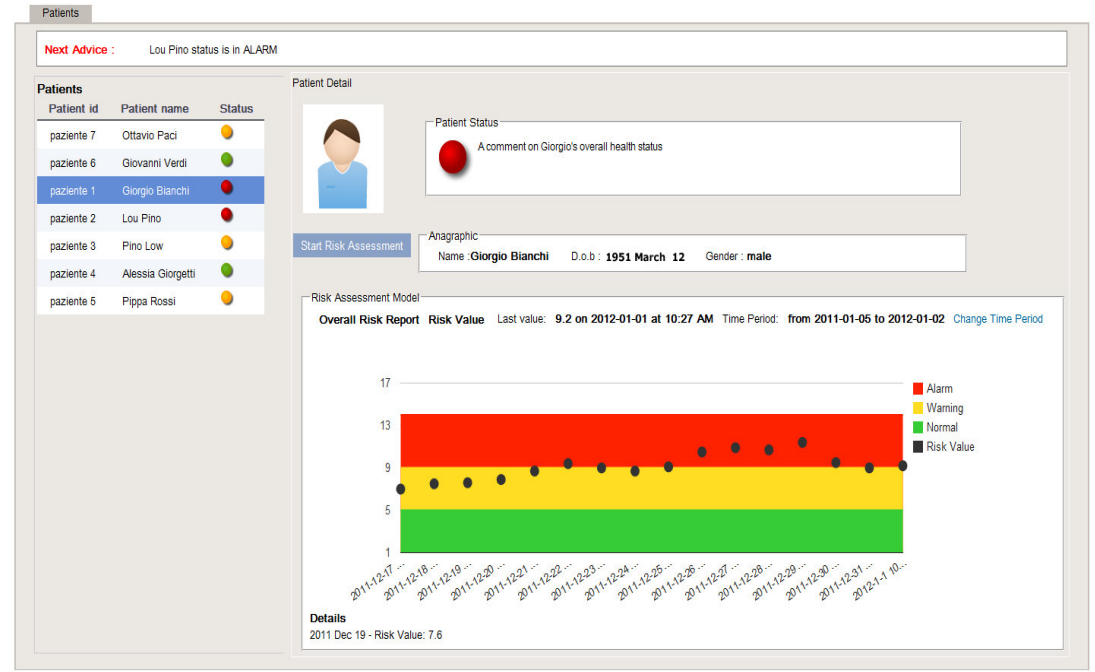

Fig. 4. Abnormal Situation Catching

The evaluation of this factor is performed automatically by the system basing on patient's profile (e.g. configured thresholds and parameters) and clinical data. Upon selection by the clinician, the system shows a more detailed view of the patient; this view includes patient's anagraphic data, and a (configurable) number of recent-past evaluations of the patient's risk factor. This last set of data is presented in a graphical way that highlights the overall trend for the selected patient, in order to ease the clinician's task to decide whether the patient's health status needs to be assessed.

If this is the case, the system will start a new Risk Assessment procedure, whose goal is to let the clinician decide whether the selected patient needs an intervention of some kind (e.g. modify the medical treatment) or the risk factor of the patient can be considered acceptable. During the procedure, the clinician can access a very broad spectrum of information regarding the patient, ranging from the patient's history of all the causes that led to the evaluation of a risk factor at a certain point in time, to the complete history of his monitored clinical parameters, along with a large database of clinical literature related with the disease of the patient and his/her monitored clinical parameters.

\subsection{Interventions}

The risk management could require one or more interventions from the medical professional depending on the assessment of the patient's risk. The sort of intervention(s) is included by the clinician in the user interface, and it could be automatic or manual. The list of actions done reacting to the situation of the person is shown to the doctor. After the intervention is carried out, the decision process is considered completed. 
The component shows the information of the causes of the intervention. The users can insert the corresponding notes about the actions done or to be carried out. Once the intervention is made, clinicians can complete the workflow. The automatic actions (SMS, email, etc) are also visible to the doctor in order to be aware of the communications maintained with the patients.

\section{Conclusion}

The healthcare management nowadays focuses on the diagnosis of the patients suffering from chronic diseases, which means, the person has to wait until an episode in order to be treated. The Medical Expert System Tool intends to stress in the prevention, monitoring the vital signs and evaluating the results comparing them to some computed profile coming from Decision Support modules.

The tool has been developed using an open and scalable design, receiving the results in a standard way. This way, different components could be applied depending on the patient's chronic disease to be monitored, because the parameters, their thresholds, rules and feedbacks are configured by the clinicians at the beginning of the workflow which guides the medical professional in the individual's follow-up.

The system allows the doctors to estimate the patients' status, positioning them in the tri-color flag (green, yellow and red). With a quick look the clinician can understand the values of the parameters studied and the possible risk factors.

More and more people trust the new technologies and tools have been proved to have the ability of "foreseeing the future" in certain cases. "Not seeing, still we know / Not knowing, guess / Not guessing, smile and hide".

Acknowledgments. This research described in this paper was carried out in the CHIRON project (Cyclic and person-centric Health management: Integrated appRoach for hOme, mobile and clinical eNvironments). The project is co-funded by the ARTEMIS Joint Undertaking (grant agreement \# 2009-1-100228) and by national authorities.

\section{References}

1. http://www.who.int/topics/chronic diseases/en/

2. Leslie SJ, Hartswood M, Meurig C, McKee SP, Slack R, Procter R, Denvir MA. Clinical decision support software for management of chronic heart failure: development and evaluation. In: Comput Biol Med. 2006 May;36(5):495-506. Epub 2005 May 31.

3. EN13606 - a Standard for EHR System Communication

4. http://qrisk.org/

5. http://www.arriba-hausarzt.de/

6. ISO 9241-210:2010 Ergonomics of human-system interaction - Part 210: Human-centred design for interactive systems 